\title{
Optimal time for remote ischemic preconditioning for liver protection in experimental major liver resection
}

\author{
(1) Ümit Alakuş ${ }^{1}$, (1) Mehmet F. Can², (1) Fevzi Nuri Aydın³, (1) Muharrem Öztaş ${ }^{1}$, (1) İsmail H. Özerhan², \\ (1) Talha Sarıgöz ${ }^{4}$, (1) Abdurrahman Şimşek ${ }^{1}$, (1) Yusuf Peker ${ }^{1}$
}

\author{
1 University of Health Sciences Turkey, Gülhane Training and Research Hospital, Clinic of General Surgery, Division of \\ Gastrointestinal Surgery, Ankara, Turkey \\ 2Lokman Hekim University Hospital, Clinic of General Surgery, Ankara, Turkey \\ ${ }^{3}$ Diskapi Yildirim Beyazit Training and Research Hospital, Clinic of Biochemistry, Ankara, Turkey \\ ${ }^{4}$ Kayseri City Hospital, Clinic of General Surgery, Kayseri, Turkey
}

Date submitted:

17.09.2020

Date accepted:

07.12.2020

Online publication date: 15.06.2021

\section{Corresponding Author:}

Ümit Alakuş, M.D., University of Health Sciences Turkey, Gülhane Training and Research Hospital, Clinic of General Surgery, Division of Gastrointestinal Surgery, Ankara, Turkey

ORCID:

orcid.org/0000-0002-6756-3583

Keywords: Remote ischemic preconditioning, liver surgery, ischemia-reperfusion injury

\begin{abstract}
Aims: Due to the liver's rich blood supply, the control of inflow is mandatory if major resection is being planned. In this regard, temporary portal triad clamping is widely used. However, ischemia-reperfusion injury (IRI) in the liver is inevitable following interruption. Remote ischemic preconditioning (IPC) was shown to ameliorate injury in the target organ in various animal models. This experimental animal study was conducted to determine optimal preconditioning frequency to use in liver surgery that promotes potential mediators associated with protection.

Methods: Male Wistar-Albino rats $(n=72)$ were divided into 8 groups. In group 1 (sham), only the left lobe of the liver was resected without any preconditioning. In group 2, the left lobe of the liver was resected using $30 \mathrm{~min}$ of portal triad clamping. In other groups, prior to resection using the same procedure with group 2,6 different remote IPC protocols ( 5 to $10 \mathrm{~min}$ ischemia plus 10 to 20 min reperfusion) were applied by clamping the femoral artery. Serum tumor necrosis factor- $\alpha$ (TNF- $\alpha$ ) and interleukin- 6 (IL-6) level analysis, liver function tests, and histological examination were performed using the TUNEL staining to study apoptosis.
\end{abstract}

Results: Compared to group 2, serum serum TNF- $\alpha$ and IL-6 level analysis and liver function tests did not show any difference. There was also no difference between the intervention groups and controls in histopathologic examination and apoptotic cell counts.

Conclusions: Remote IPC protocols we studied in this experiment did not blunt hepatic IRI in rodents.

\section{Introduction}

Due to the liver's rich blood supply, the control of inflow is mandatory if major resection is being planned. Temporary portal triad clamping is used widely to reduce bleeding during transection of the liver, but ischemia and subsequent reperfusion causes injury and direct cell damage through complicated inflammatory events $(1,2)$.

The proposal of ischemic preconditioning (IPC) was first made by Murry et al. $(3,4)$ following their experience with dog myocardium. They postulated that brief episodes of ischemia could prepare the target tissue for sustained ischemic attack. Later, this concept was adapted to the liver surgery. However, direct IPC by the occlusion of portal inflow was shown to disrupt regeneration capacity of the liver $(5,6)$.

The idea was worked up further by Przyklenk et al. (7) in canines and they reported that remote IPC at a distant vascular bed could result in decreased target organ injury after sustained occlusion. Various studies have investigated the role of remote 
IPC in several organs including the liver $(8,9)$. However, there is no consensus on the mechanism responsible and the optimal timing of the method in the setting of liver surgery (10).

In this study, based on reported benefits of remote IPC in the target organ, it was postulated that by using remote IPC, we could prepare liver tissue for further ischemia-reperfusion injury which is an inevitable result of portal triad clamping. The aim of this experimental animal study was to determine and optimize the ideal time for remote IPC that would protect liver function and histomorphology the best after liver IRI in rats. In this regard, six different remote IPC procedures were modeled and we sought the potential benefit of remote IPC during liver resection in an animal model. Remote IPC was applied after the isolation of the right femoral artery rather than limb tourniquet which provided precise ischemia.

\section{Methods}

\section{Animal Experiments}

The study was conducted at Gülhane Military of Academy of Medicine with the approval of Gülhane Military of Academy of Medicine Animal Studies Ethics Committee numbered 201141-11/48 and in accordance with the Guide for the Care and Use of Laboratory Animals (National Research Council Institute for Laboratory Animal Research, US, Washington, National Academies Press, 1996).

Before the experiment, sample size was determined to be 72 to have $80 \%$ power and to demonstrate statistical difference between the planned protocols. The effect size of this experimental animal study was 0.5 and the significance level was accepted at 0.05 .

Seventy-two one-month-old Wistar Albino rats weighed $315 \pm 35$ gram were used. The rats were held in metal cages, fed with standard laboratory chow diet and watered with ambient temperature at $22 \pm 2{ }^{\circ} \mathrm{C}$. They were randomized into 8 groups (Table 1).

\section{Anesthesia Induction}

They were fasted for 12 hours before surgery. General anesthesia was maintained with Ketamine (Ketalar ${ }^{\circledR}$, Parke
Davis and Co. Inc., 40 mg/kg) and Xylazin (Rompun ${ }^{\circledR}$, Bayer Ag, Leverkusen, Germany; $5 \mathrm{mg} / \mathrm{kg}$ ).

\section{Surgical Procedure}

The rats were placed in supine position. The procedures were performed by a surgeon for standardization in accordance with the principles of asepsis and antisepsis. Abdominal area was incised in a bilateral subcostal fashion and if remote IPC was being planned, right inguinal region was cut vertically over the femoral artery trace. The method of remote IPC was carried out by dissecting the femoral artery, vein and nerve. The femoral artery was isolated and occluded with a microvascular clamp (Vasculostatt-Scanlan, St Luis, USA). The time of occlusion and reperfusion periods varied according to the study groups. After the completion of IPC, the inguinal incisions were sutured with 3/0 polypropylene (Figure 1).

Liver exploration was performed through a bilateral subcostal incision. First, lobar anatomy of the rats was explored. Falciform ligament was cut. Hepatoduodenal ligament was dissected if Pringle's maneuver was planned. For the maneuver, microvascular clamp was used and hepatoduodenal ligament was occluded for 30 minutes. The pedicle of left lateral section was found, tied with 5/0 silk, and cut. Later, connections of the left medial lobe were tied and cut. With the resection of the left lateral lobe and left medial lobe, nearly $40 \%$ of the liver was resected. Following 30 minutes of total occlusion, clamp was removed and reperfusion was initiated. The bilateral subcostal incision was closed using 3/0 polypropylene (Table 1, Figure 2).

In group 1, after the completion of resection and in the other groups, following $180^{\text {th }}$ minute after the removal of the portal clamp, the rats were explored again. For the biochemical analysis, intracardiac blood samples were obtained and for the histopathological evaluation, remnant liver tissue was resected. The rats were sacrificed.

The blood samples were centrifuged for 10 minutes at $3.000 \mathrm{Xg}$ and the serum was stored at - 80-degree Celsius. The remnant liver tissue was fixed in $10 \%$ formaldehyde. The changes in liver histomorphology was evaluated under light microscope with hematoxylin-eosin (H\&E) staining and terminal

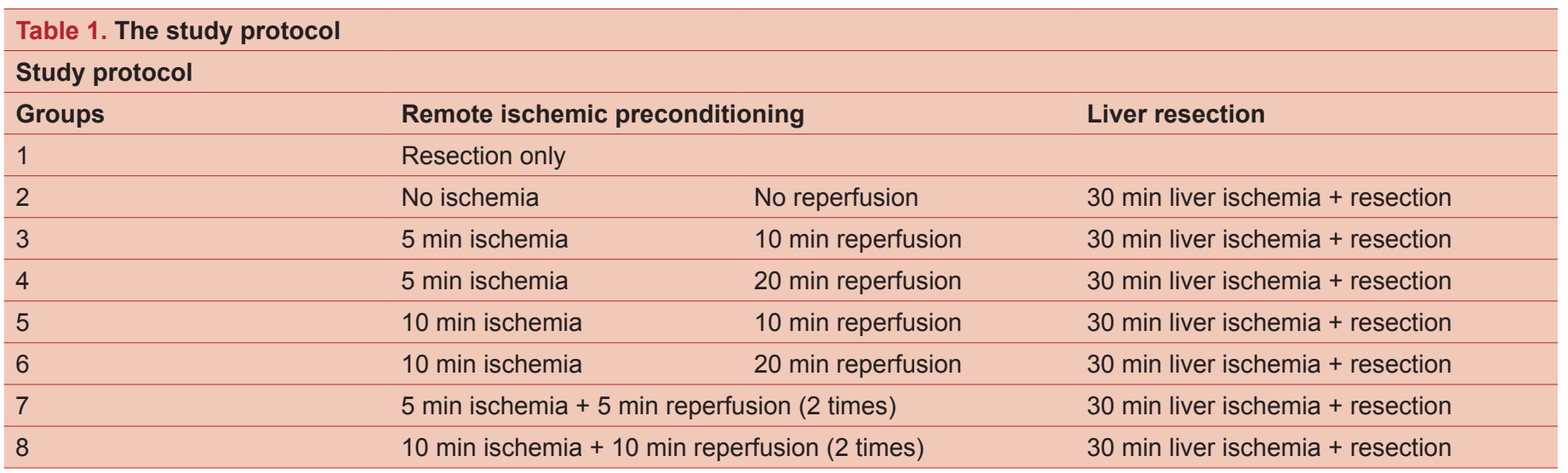




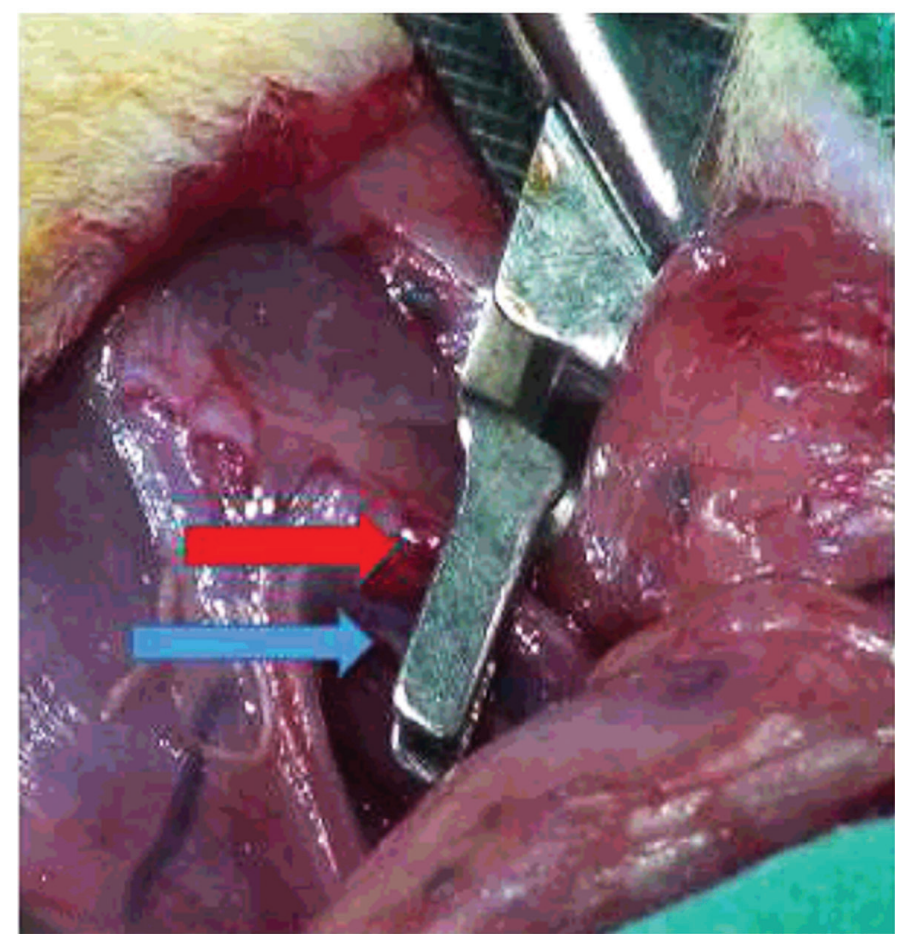

Figure 1. Clamping of femoral artery for remote ischemic preconditioning protocol

Red arrow: femoral artery; blue arrow: femoral vein

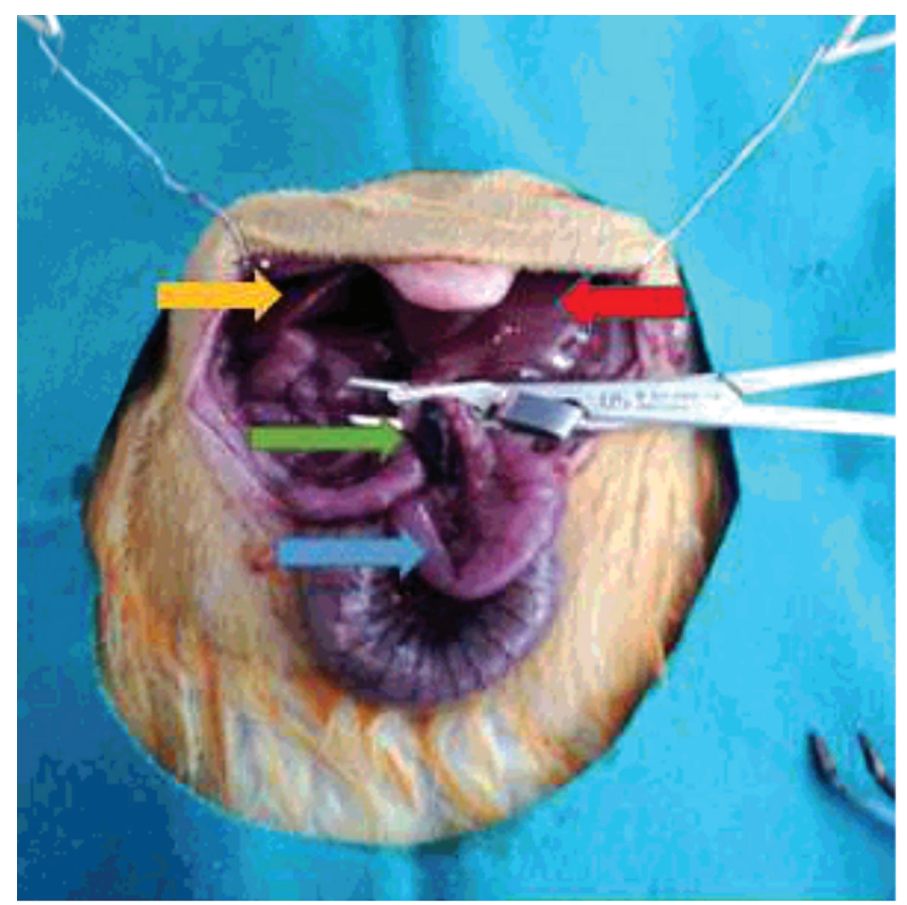

Figure 2. Pringle maneuver (hepatoduodenal ligament occlusion)

Yellow arrow: liver (right lobe); red arrow: liver (left lobe); green arrow: hepatoduodenal ligament; blue arrow: intestines

deoxynucleotidyl transferase dUTP nick end labeling (TUNEL) assay. The degree of liver injury and apoptosis levels were recorded for statistical analysis by a pathologist blinded to the study.

\section{Biochemical Analysis}

\section{Serum TNF- $\alpha$ and IL-6 Levels}

From the serum samples, tumor necrosis factor- $\alpha$ (TNF- $\alpha$ ), interleukin-6 (IL-6), aspartate aminotransferase (AST), alanine aminotransferase (ALT), lactate dehydrogenase (LDH), total bilirubin (TB), alkaline phosphatase (ALP), and gamma glutamyl transferase (GGT) levels were calculated. Calculation of serum TNF- $\alpha$ and IL-6 levels were made using enzyme-linked immunosorbent assay kits and according to the producer's recommendation (eBioscience, Vien, Austria, catalog number BMS622, BMS625) in Sinergy Plate Reader (Bio-Tek Instruments Inc, Winooski, VT, USA) at 450 um.

\section{Liver Enzymes}

Serum transferases, LDH, TB, ALP and GGT were measured with a standard spectrophotometric method with an automated clinical analyzer (JCA-BM9030; JEOL, Ltd., Tokyo, Japan).

\section{Histopathological Evaluation}

Following reperfusion, at the $3^{\text {rd }}$ hour, remnant liver tissue was resected and fixed. After the dehydration process, tissue was embedded in paraffin blocks. The sections were cut at $4 \mu \mathrm{m}$ thickness and the liver structure was examined by H\&E stain and TUNEL assay.

\section{Hematoxylin-eosin Staining}

The sections were evaluated under light microscope for determining the degree of liver injury using H\&E stain. A modified example of the scale which was designed by Camargo et al. (11) was used (Grade 0: Minimal or no evidence of injury, Grade 1: Cytoplasmic vacuolation or mild injury with focal nuclear pyknosis; Grade 2: Moderate injury characterized by cytoplasmic vacuolation, hepatocyte ballooning degeneration, sinusoidal dilatation and congestion, loss of intercellular borders; Grade 3: Moderate injury characterized by areas of coagulation necrosis, cytoplasmic hypereosinophilia, wide sinusoidal dilatation and congestion; Grade 4: Severe coagulation necrosis with disintegration of hepatic cords, hemorrhage).

\section{TUNEL Assay}

Each section was evaluated by an expert pathologist blinded to the study. Apoptotic index was calculated as Lai et al. (12) used in their study using the proportion of positive stained cells to total cells in 5 different areas and the degree of apoptosis was scored as 1 for $1-33 \%, 2$ for $34-66 \%$, and 3 for $67-100 \%$.

\section{Statistical Analysis}

For the statistical analysis, Statistical Package for Social Sciences software (SPSS version 15.0, SPSS Inc., Chicago, IL, USA) was used. The data were presented using number, percentage, mean, standard deviation, median, minimum and maximum values. The chi-squared test and the Fisher's Exact 
test were used to compare the batch process data. For the analysis of continuous variables without normal distribution, the Mann-Whitney $U$ test was used.

\section{Results}

\section{Serum TNF- $\alpha$ Levels}

Regarding the serum TNF- $\alpha$, significantly lower values were observed in group 1 compared to group 2. When group 2 and the IPC groups were evaluated, group 3, 4 and 8 were found to have significantly lower levels $(p<0.05)$. On the other hand, group 6 and 7 had also lower levels of serum TNF- $\alpha$, but this difference was not significant ( $p>0.05)$.

\section{Serum IL-6 Levels}

Although observed levels of IL-6 were lower in group 1, there was not significant difference between group 1 and group 2 ( $p>0.05)$. When group 2 was compared to the other groups, significant decrease was not observed in the IPC groups.

\section{Total Bilirubin}

There was not significant difference between group 1 and group 2 considering TB levels $(p>0.05)$. When group 2 was compared to the other groups, significant difference was not observed $(p>0.05)$.

\section{Liver Enzymes}

In comparison to group 2, lower levels of AST and ALT were observed in group 1 but this difference was not significant $(p>0.05)$. In addition, there was not significant difference between the IPC groups and group 2 with regard to AST and ALT. Concerning ALP, GGT and LDH, statistically significant difference was not observed among the groups.

\section{Hematoxylin-eosin Staining}

The sections were evaluated under light microscope by a pathologist blinded to the study in terms of the degree of liver injury. Neither grade 3 nor grade 4 injury was observed. Between group 1 and group 2, there was not statistically significant difference regarding H\&E staining ( $p>0.05$ ) (Table 2). In both groups, similar rate of liver injury was observed. When group 2 was compared to the groups with IPC protocol, the degree of general histopathological liver injury did not differ among the groups. Between-group comparisons showed no statistically

Table 2. The comparison of group 1 and group 2 with regard to hematoxylin-eosin staining

\begin{tabular}{|c|c|c|c|}
\hline & n (\%) & & $\mathbf{p}^{*}$ \\
\hline H\&E & Group 1 & Group 2 & \multirow{3}{*}{1.000} \\
\hline Grade 0 & $4(44.4)$ & $3(33.3)$ & \\
\hline Grade 1 & $5(55.5)$ & $6(66.6)$ & \\
\hline $\begin{array}{l}\text { *Fisher's Exact test. } \\
\text { H\&E: Hematoxylin-eosin }\end{array}$ & & & \\
\hline
\end{tabular}

significant decrease in liver injury scores in the groups with IPC protocol (group 2 vs. group 3, $p=0.153$; group 2 vs. group 4, $p=0.150$; group 2 vs. group $5, p=1.000$; group 2 vs. group 6 , $p=1.000$; group 2 vs. group $7, p=0.354$; group 2 vs. group 8 , $\mathrm{p}=0.288$ ).

\section{TUNEL Assay}

The apoptotic index result in group 1 was found to be significantly lower than group $2(p<0.05)$. When group 1 was compared to the other groups with preconditioning method, the apoptosis index was detected to be lower in group $1(p<0.05)$. There was not statistically significant difference between group 2 and the other groups that IPC was applied (group 2 vs. group $3, p=0.569$; group 2 vs. group $4, p=0.516$; group 2 vs. group 5, $\mathrm{p}=0.219$; group 2 vs. group $6, p=0.514$; group 2 vs. group 7 , $p=0.630$; group 2 vs. group $8, p=0.282$ ). Remote IPC was not found to decrease apoptotic IRI in the liver (Table 3).

\begin{tabular}{|c|c|c|c|}
\hline & n (\%) & & $\mathbf{p}^{*}$ \\
\hline TUNEL staining & Group 1 & Group 2 & \multirow{4}{*}{0.002} \\
\hline $0 \%$ & $9(100)$ & $2(22.2)$ & \\
\hline $1-33 \%$ & $0(0)$ & $6(66.6)$ & \\
\hline $34-66 \%$ & $0(0)$ & $1(11.1)$ & \\
\hline
\end{tabular}

\section{Discussion}

Recent progress in the liver surgery has led to the wide range of methods available for resection. The concept of remote IPC was first described by Przyklenk et al. (7) in canine heart model. Due to doubtful ischemic periods and direct trauma to vascular structures, clinic application is limited (9). Considering the reported protective effects of remote IPC, potential benefits and various cycles were investigated with the animal model of liver resection in this study. Six remote IPC methods, which were all differentiated in terms of time interval, were employed in the light of the literature. Nonetheless, we were not able to demonstrate significant improvement in postoperative liver injury following partial liver resection, which is largely in agreement with the previous studies from different authors. None of the proposed remote IPC methods provided potential benefit in liver resection.

Although introduced in 1908, the Pringle's (13) maneuver is yet the most common applied technique to reduce bleeding and associated complications during parenchymal liver resections. It is based on total occlusion of hepatic pedicle. As a result of that application, IRI in the remnant liver tissue is still a problem awaiting for solution. The remote IPC method was shown to be beneficial for reducing IRI in the heart, kidney and lung $(14,15)$. In 1986, Murry et al. $(3,4)$ studied the effects of repeated brief 
episodes of IPC, either single or multiple, on canine myocardium and reported salutary results with respect to sustained ischemia. In the following years, Przyklenk et al. (7) carried this idea a bit further and defined remote IPC. They indicated that brief episodes of remote IPC protected remote virgin myocardial tissue from sustained ischemic insult. These studies have revealed that IPC of an organ reduces the degree of injury in the target tissue during continuous ischemia by unknown mechanisms (9). In their review, Robertson et al. (16) have indicated that the effects of remote IPC on IRI partially relies on the release of adenosine and L-arginine. In another rodent study, Oberkofler et al. (17) identified a platelet-dependent axis that could mitigate hepatic IRI.

Impact of remote IPC on the liver was evaluated by Kanoria et al. (9) in an experimental rabbit study. In this study, the right hind legs of rabbits were subjected to 3 cycles of 10-min ischemia and reperfusion. They observed significant difference after remote IPC in aminotransferase levels, peripheric oxygen saturation and hepatic blood flow. In the work of Guimarães Filho et al. (18), 6 cycles of 4-min ischemia and 4-min reperfusion on the right hind limb were employed. While plasmatic ALT levels were significantly lower, IL-6 levels were significantly higher in the remote IPC group. Also, there was a clear reduction in necrosis in the remote IPC group histopathological evaluation. In this study, we did not observe significant changes in $\mathrm{LDH}$, ALP, TB, GGT and IL-6 levels among the groups. Although aminotransferases were lower in group 1 , statistically significant results were not observed. Although again reperfusion times were similar to Kanoria et al. (9) and Guimarães Filho et al.'s (18) study, we contributed unexpected insignificant results to the remote IPC method we used and partial liver resection procedure because there was no other difference between their model and this study. Liver resection might have caused additional trauma. In another study, Jia et al. (15) investigated the impact of remote IPC on the liver grafts and concluded that 3 cycles of 5-min remote IPC seemed to be more efficacious during liver transplant. However, they applied remote IPC on both of the hindlimbs and they did not use portal triad clamping as in our study. As an alternative method, Kageyama et al. (19) applied intestinal remote IPC consisting of 2 four-minute superior mesenteric artery clamping separated by 11-minute declamping. In their model, it was shown that the intestines could also be a target for remote IPC. Furthermore, release of serum transaminases, LDH, IL-6 and TNF- $\alpha$ were decreased in the intestinal remote IPC group compared to the sham group.

On the other hand, in this work up, we observed significantly lower levels of TNF- $\alpha$ in group 3, 4 and 8 . In these groups, 5-min ischemia and 10-min reperfusion; 5-min ischemia and 20-min reperfusion; 2 cycles of 10-min ischemia and 10-min reperfusion were applied, respectively. However, we could not find clear explanation for lower levels of TNF- $\alpha$ in those. There was not significant difference in histopathological examination. Furthermore, in the aforementioned studies, remote IPC was induced using limb tourniquet or bundle occlusion but in this rodent trial, only femoral artery was clamped subsequent to detailed dissection because it was postulated that selective blockage of the femoral artery could overcome possible inadequate blood occlusion and nerve paralysis due to the use of tourniquet. Nevertheless, we associated the unsatisfactory results with this choice of technique. In the study of Zhang et al. (20), they also attributed dissatisfying results to the dissection of the femoral artery and vein rather than limb ischemia. Limb ischemia could cause stress on skeletal muscles and might induce an unknown interaction between target organ and muscle $(21,22)$. Further studies should be planned to clarify this mechanism. Apart from the method of remote IPC, this study also differentiated from the similar ones as left lobectomy was performed. Most of the others collected samples after the completion of IPC. Actually, we aimed to simulate clinical practice but we concluded that this procedure might have negative effect on remnant liver and it could be the reason behind unsatisfactory results. In a different study, Gustafsson et al. (23) clamped the femoral artery of Wistar rats to induce remote IPC and also could not demonstrate its beneficial effect. Their explanation was that short period of IPC, which was 10 minutes, caused inadequate effect. Considering all the above-discussed factors, investigated remote IPC protocols were not enough to blunt hepatic IRI in this rodent trial.

The experience in animal models has been translated to clinical practice, as well. The first clinical trial was conducted by Clavien et al. (24), who studied the effect of remote IPC with a cycle of 10-min ischemia and 10-min reperfusion on the remnant liver. Promising results, which were indicated by lower serum transferase levels, were achieved. In the recent pilot study of Kanoria et al. (25), they studied the outcome of remote IPC, which was induced through three 10-min cycles, in patients undergoing major liver resection due to colorectal cancer metastasis. They were able to show protective effects of remote IPC and safety of tourniquet application. Robertson et al. (26) studied with transplant recipients. They induced remote IPC using limb tourniquet through three 5-min cycles and reported that there was no evidence of decrease regarding IRI in the short-term but long-term studies are needed. Also, these studies have shown that limb tourniquet is a safe procedure to employ remote IPC. In the systematic review of Farooqui et al. (27), 12 studies were included and it was concluded that the translation of remote IPC to clinical practice should be considered with caution due to heterogeneity of populations and preconditioning set-ups. Therefore, the remote IPC protocols should be discussed widely for future patients who will greatly benefit from this preconditioning. 
Our study has some limitations. It can be evaluated that the liver ischemia of 30 minutes we applied may be an insufficient time to demonstrate the effectiveness of remote IPC. In the literature, protective effect of remote IPC by inducing liver ischemia for a longer time has been shown in studies.

However, several other researchers used partial clamping techniques. In our study, unlike other studies, the results of liver resection can be thought to have negative effect. However, as is known, the Pringle maneuver is a bleeding reducer that is often required during liver resection. Our efforts to include liver resection in the study aimed to simulate the clinical practice and scenario encountered correctly. Another limitation in our study is that we did not evaluate the late effects of the remote IPC. However, in our opinion, the demonstration of biochemical and histopathological damage and TUNEL method demonstration of apoptosis is sufficient to examine the subject procedure (28).

\section{Conclusion}

In conclusion, since the description of remote IPC, it has been a focus of extensive research. However, there is still no clear explanation of a mechanism. While there are various factors affecting outcomes such as heterogenous participants, different methods of IPC and application site, none of the trials has been successful in showing any benefit of remote IPC to reduce IRI or clinically relevant consequences. Yet, it is still worth considering as a potential method for reducing hepatic IRI during liver surgery.

\section{Acknowledgements}

We want to thank deceased "Yusuf Peker" who was the head of the department when the study took place for his support. He died on 5 September 2015.

\section{Ethics}

Ethics Committee Approval: The study was conducted at Gülhane Military of Academy of Medicine with approval of Gülhane Military of Academy of Medicine Animal Studies Ethics Committee (no: 2011-41-11/48) and in accordance with Guide for the Care and Use of Laboratory Animals (National Research Council Institute for Laboratory Animal Research, US, Washington, National Academies Press, 1996).

Informed Consent: This was an animal study.

Peer-review: Externally peer-reviewed.

\section{Authorship Contributions}

Surgical and Medical Practices: Ü.A., M.F.C., F.N.A., M.Ö., I.H.Ö., A.Ş., Y.P., Concept: Ü.A., M.F.C., F.N.A., M.Ö., İ.H.Ö., T.S., A.Ş., Y.P., Design: Ü.A., M.F.C., M.Ö., I.H.Ö., T.S., A.Ş., Y.P., Data Collection or Processing: Ü.A., M.F.C., F.N.A., M.Ö., I.H.Ö., T.S., Analysis or Interpretation: Ü.A., M.F.C., F.N.A.,
M.Ö., I.H.Ö., T.S., Literature Search: Ü.A., M.F.C., M.Ö., I.H.Ö., T.S., A.Ş., Y.P., Writing: Ü.A., M.F.C., M.Ö., I.H.Ö., T.S., A.Ş., Y.P.

Conflict of Interest: No conflict of interest was declared by the authors.

Financial Disclosure: The authors declared that this study received no financial support.

\section{References}

1. Kim YI. Ischemia-reperfusion injury of the human liver during hepatic resection. J Hepatobiliary Pancreat Surg. 2003;10:195-199.

2. Jaeschke H. Molecular mechanisms of hepatic ischemiareperfusion injury and preconditioning. Am J Physiol Gastrointest Liver Physiol. 2003;284:G15-G26.

3. Reimer KA, Murry CE, Yamasawa I, Hill ML, Jennings RB. Four brief periods of myocardial ischemia cause no cumulative ATP loss or necrosis. Am J Physiol Heart Circ. 1986;251:H1306-H1315.

4. Murry CE, Jennings RB, Reimer KA. Preconditioning with ischemia: a delay of lethal cell injury in ischemic myocardium. Circulation. 1986;74:1124-1136.

5. Yao A, Li X, Pu L, et al. Impaired hepatic regeneration by ischemic preconditioning in a rat model of small-for-size liver transplantation. Transpl Immunol. 2007;18:37-43.

6. Eipel C, Glanemann M, Nuessler AK, Menger MD, Neuhaus P, Vollmar B. Ischemic preconditioning impairs liver regeneration in extended reduced-size livers. Ann Surg. 2005;241:477-484.

7. Przyklenk K, Bauer B, Ovize M, Kloner RA, Whittaker $P$. Regional ischemic 'preconditioning' protects remote virgin myocardium from subsequent sustained coronary occlusion. Circulation. 1993;87:893-899.

8. Kharbanda RK, Mortensen UM, White PA, et al. Transient limb ischemia induces remote ischemic preconditioning in vivo. Circulation. 2002;106:2881-2883.

9. Kanoria S, Jalan R, Davies NA, Seifalian AM, Williams R, Davidson BR. Remote ischaemic preconditioning of the hind limb reduces experimental liver warm ischaemiareperfusion injury. Br J Surg. 2006;93:762-768.

10. Magyar Z, Varga G, Mester A, et al. Is the early or delayed remote ischemic preconditioning the more effective from a microcirculatory and histological point of view in a rat model of partial liver ischemia-reperfusion? Acta Cirurgica Brasileira. 2018;33:597-608.

11. Camargo CA Jr, Madden JF, Gao W, Selvan RS, Clavien PA. Interleukin-6 protects liver against warm ischemia/ reperfusion injury and promotes hepatocyte proliferation in the rodent. Hepatology. 1997;26:1513-1520.

12. Lai IR, Chang KJ, Tsai HW, Chen CF. Pharmacological preconditioning with simvastatin protects liver from 
ischemia-reperfusion injury by heme oxygenase-1 induction. Transplantation. 2008;85:732-738.

13. Pringle $\mathrm{JH}$. V. Notes on the arrest of hepatic hemorrhage due to trauma. Ann Surg. 1908;48:541-549.

14. Yadav SS, Sindram D, Perry DK, Clavien PA. Ischemic preconditioning protects the mouse liver by inhibition of apoptosis through a caspase-dependent pathway. Hepatology. 1999;30:1223-1231.

15. Jia J, Li J, Jiang L, et al. Protective effect of remote limb ischemic perconditioning on the liver grafts of rats with a novel model. PLoS One. 2015;10:e0121972.

16. Robertson F, Fuller B, Davidson B. An evaluation of ischaemic preconditioning as a method of reducing ischaemia reperfusion injury in liver surgery and transplantation. J Clin Med. 2017;6:69.

17. Oberkofler CE, Limani $\mathrm{P}$, Jang JH, et al. Systemic protection through remote ischemic preconditioning is spread by platelet-dependent signaling in mice. Hepatology. 2014;60:1409-1417.

18. Guimarães Filho MAC, Cortez E, Garcia-Souza ÉP, et al. Effect of remote ischemic preconditioning in the expression of IL-6 and IL-10 in a rat model of liver ischemia-reperfusion injury. Acta Cir Bras. 2015;30:452-460.

19. Kageyama $\mathrm{S}$, Hata $\mathrm{K}$, Tanaka $\mathrm{H}$, et al. Intestinal ischemic preconditioning ameliorates hepatic ischemia/reperfusion injury in rats: Role of heme oxygenase 1 in the second window of protection. Liver Transplant. 2015;21:112-122.

20. Zhang Y, Nakano D, Nishiyama A. Remote ischemic preconditioning of the femoral artery and vein does not protect against renal ischemia/reperfusion-induced injury in anesthetized mice. J Urol Nephrol Open Access. 2016;2:1-5
21. Pavyde E, Maciulaitis R, Mauricas M, et al. Skeletal muscle-derived stem/progenitor cells: a potential strategy for the treatment of acute kidney injury. Stem Cells Int. 2016;2016:9618480.

22. Mclntire KL, Chen Y, Sood S, Rabkin R. Acute uremia suppresses leucine-induced signal transduction in skeletal muscle. Kidney Int. 2014;85:374-382.

23. Gustafsson BI, Friman S, Wallin M, Heiman J, Delbro DS. Effect of remote preconditioning on mild or severe ischemia-reperfusion injury to rat liver. Transplant Proc. 2006;38:2708-2709.

24. Clavien PA, Yadav S, Sindram D, Bentley RC. Protective effects of ischemic preconditioning for liver resection performed under inflow occlusion in humans. Ann Surg. 2000;232:155-162.

25. Kanoria S, Robertson FP, Mehta NN, Fusai G, Sharma D, Davidson BR. Effect of remote ischaemic preconditioning on liver injury in patients undergoing major hepatectomy for colorectal liver metastasis: a pilot randomised controlled feasibility trial. World J Surg. 2016;41:1322-1330.

26. Robertson FP, Goswami R, Wright GP, et al. Remote ischaemic preconditioning in orthotopic liver transplantation (RIPCOLT trial): a pilot randomized controlled feasibility study. HPB (Oxford). 2017;19:757-767.

27. Farooqui W, Pommergaard HC, Rasmussen A. Remote ischemic preconditioning of transplant recipients to reduce graft ischemia and reperfusion injuries: a systematic review. Transplant Rev (Orlando). 2018;32:10-15.

28. Wang F, Birch SE, He R, et al. Remote ischemic preconditioning by hindlimb occlusion prevents liver ischemic/reperfusion injury: the role of High Mobility GroupBox 1. Ann Surg. 2010;251:292-299. 